\title{
UPDATE ON DEFINITIONS OF SARCOPENIA
}

\author{
S. STUDENSKI
}

Longitudinal Studies Section, Intramural Program, National Institute on Aging, Baltimore, MD, USA

Corresponding author: Stephanie Studenski, Longitudinal Studies Section, Intramural Program, National Institute on Aging, Baltimore, MD, USA, Phone: +1 (410) 350-3964, Email: stephanie.studenski@nih.gov

J Frailty Aging 2015;4(4):173-174

Published online June 23, 2015, http:/ / dx.doi.org/10.14283/jfa.2015.60

\section{Introduction}

Although the age-related loss of muscle mass has been widely recognized as a significant public health problem throughout the world, the absence of a consensus definition has limited diagnosis and treatment of the condition and slowed the development of new and effective interventions. Many definitions and diagnostic criteria have been proposed, reflecting similar key concepts although differing in details (1-4). A gold standard, evidence-based definition of clinically important weakness and low muscle mass is clearly needed both for clinical use and to advance clinical research.

\section{Defining sarcopenia}

Sarcopenia was originally defined as low muscle mass associated with aging (5), with the assumption that low mass causes functional problems due to weakness. However, muscle mass does not correlate well with strength: many people have low muscle mass but are strong, while many others are weak despite having normal muscle mass. Muscle quality - defined as reduced force produced per unit of mass -differentiates these two groups of people. Some have proposed that sarcopenia should refer to the combination of low muscle mass with weakness, recognizing that weakness may be more important clinically than mass. In 2008, Manini and Clark coined the term "dynapenia" to more accurately describe the age-related loss of muscle strength (6).

Reduced muscle mass and weakness may result from underlying disease, disuse, or as a result of aging. For example, cachexia refers to the accelerated wasting of muscle that occurs in persons with cancer, and is thought to involve inflammation. Age-related sarcopenia probably results from a combination of inflammatory mechanisms and disuse. The European Working Group on Sarcopenia in Older People (EWGSOP) proposed classifying sarcopenia as primary sarcopenia if related to aging alone, and secondary if resulting from an underlying disease, nutritional deficiency, or disuse from extended bed rest or a sedentary lifestyle (1). Anker and colleagues proposed unifying the concepts of sarcopenia and cachexia under the umbrella term "muscle wasting disease," further classifying conditions according to etiology, severity, and progression (7).

In 2010, the Foundation for the National Institute of Health (FNIH) Biomarkers Consortium launched the Sarcopenia Project to develop data-driven diagnostic criteria for the condition. The project brought together experts from the National Institute on Aging (NIA), the Food and Drug Administration (FDA), several academic research institutions, industry partners, and a non-for-profit organization. At a conference in 2012, they recommended further analyses to define criteria for weakness and, low muscle mass, as well as longitudinal predictions based on these criteria. To accomplish this, cross-sectional analyses were conducted using data from nine collaborating studies encompassing 26,625 older, community-dwelling adults. These analyses were published in a series of articles in 2014 (8-12).

\section{Beyond the recommended diagnostic criteria}

The FNIH Sarcopenia project recommended cutpoints for weakness and low lean mass in men and women, as well as further studies to better understand gender differences and how body composition relates to function. Different cutpoints for men and women may, by themselves, be insufficient; different assessment strategies may also be needed. For example, approaches that adjust for body mass index or body fat seem to be especially important for obtaining accurate assessments in women.

They also recommended developing criteria for muscle quality, such as force per unit of mass, and further studies to determine the relationship of muscle quality to mobility limitations and disability. Data from the Baltimore Longitudinal Study of Aging (BLSA) has shown that declining muscle quality is associated with higher levels of fat body mass and lower levels of lean body mass, and that it declines much faster in women than in men (in press). Knee extension peak torque at 60 seconds, adjusted for thigh cross sectional area provides a measure of muscle quality over time, but other simpler measures of strength and appendicular lean body mass, such as grip strength adjusted for body mass index (BMI), may provide indicators of muscle quality that are as good or better in terms of discriminating function.

To expedite clinical trials and better understand the predictive ability of various outcomes, a standard set of outcome measures will be proposed, including performance measures, patient-reported outcomes, assessments of injury and disability, and health care utilization.

Studies are also need to better understand the relationship between mass, strength, and function in populations with a high prevalence of mobility limitations and disability, including 


\section{UPDATE ON DEFINITIONS OF SARCOPENIA}

specific at-risk populations such as individuals who have had a hip fracture. To better understand muscle quality, it may also be useful to examine the characteristics of people who have low lean mass but superior muscle quality. These future studies along with others that assess the functional impact of improved lean mass, strength, and muscle quality should provide important information to guide intervention trials.

\section{Conflict of interest: None}

Acknowledgements: This research was supported in part by the Intramural Research Program of the National Institute on Aging.

\section{References}

1. Cruz-Jentoft AJ, Baeyens JP, Bauer JM, et al. Sarcopenia: European consensus on definition and diagnosis: Report of the European Working Group on Sarcopenia in Older People. Age Ageing. 2010;39(4):412-23.

2. Morley JE, Abbatecola AM, Argiles JM, et al. Sarcopenia with limited mobility: an international consensus. J Am Med Dir Assoc. 2011;12(6):403-9.

3. Muscaritoli M, Anker SD, Argiles J, et al. Consensus definition of sarcopenia, cachexia and pre-cachexia: joint document elaborated by Special Interest Groups (SIG) «cachexia-anorexia in chronic wasting diseases» and «nutrition in geriatrics». Clin Nutr. 2010;29(2):154-9.
4. Fielding RA, Vellas B, Evans WJ, et al. Sarcopenia: an undiagnosed condition in older adults. Current consensus definition: prevalence, etiology, and consequences. International working group on sarcopenia. J Am Med Dir Assoc. 2011;12(4):249-56.

5. Rosenberg IH. Sarcopenia: origins and clinical relevance. J Nutr. 1997;127(5 Suppl):990S-1S.

6. Clark BC, Manini TM. Sarcopenia =/= dynapenia. J Gerontol A Biol Sci Med Sci. 2008;63(8):829-34.

7. Anker SD, Coats AJ, Morley JE, et al. Muscle wasting disease: a proposal for a new disease classification. J Cachexia Sarcopenia Muscle. 2014;5(1):1-3.

8. Alley DE, Shardell MD, Peters KW, et al. Grip strength cutpoints for the identification of clinically relevant weakness. J Gerontol A Biol Sci Med Sci. 2014;69(5):559-66.

9. Cawthon PM, Peters KW, Shardell MD, et al. Cutpoints for low appendicular lean mass that identify older adults with clinically significant weakness. J Gerontol A Biol Sci Med Sci. 2014;69(5):567-75.

10. Dam TT, Peters KW, Fragala M, et al. An evidence-based comparison of operational criteria for the presence of sarcopenia. J Gerontol A Biol Sci Med Sci. 2014;69(5):584-90.

11. McLean RR, Shardell MD, Alley DE, et al. Criteria for clinically relevant weakness and low lean mass and their longitudinal association with incident mobility impairment and mortality: the foundation for the National Institutes of Health (FNIH) sarcopenia project. J Gerontol A Biol Sci Med Sci. 2014;69(5):576-83.

12. Studenski SA, Peters KW, Alley DE, et al. The FNIH sarcopenia project: rationale, study description, conference recommendations, and final estimates. J Gerontol A Biol Sci Med Sci. 2014;69(5):547-58. 